\title{
Barriers to Access to Antiretroviral Treatment in Mozambique, as Perceived by Patients and Health Workers in Urban and Rural Settings
}

\author{
Mariana Posse, M.A., and Rob Baltussen, Ph.D. ${ }^{1}$
}

\begin{abstract}
This study identifies, ranks, and compares factors perceived as barriers to accessing antiretroviral treatment (ART) in urban and rural settings in Mozambique. Data were collected between March and July 2008. It consisted of 13 focus group discussions and a structured questionnaire administered to 252 people living with HIV/AIDS (PLWHA) and 28 health workers in the districts of Beira and Buzi. Data analysis was performed using content analysis, factor analysis, and percentages of the maximum attainable scores. The data analysis revealed six clusters of factors, which were ranked according to the percentages of the maximum attainable scores between brackets: (1) patient resource availability, in which distance from home to the health facility, transportation and food availability were rated below $40 \%$; (2) community information (47\%), and (3) service availability (53\%), in which the waiting time to receive the results of CD4 analysis and the sufficiency of doctors/nurses at the health facility were both rated at $45 \%$; (4) patient information and attitudes toward treatment $(74 \%)$; family support $(77 \%)$ and health personnel confidentiality (79\%). Policy makers, in efforts to further improve the access to ART may decide to target their attention in designing interventions to improve specific aspects of patient resource availability, community information, and service availability in both urban and rural settings.
\end{abstract}

\section{Introduction}

$\mathbf{T}$ HE INTRODUCTION OF antiretroviral treatment (ART) has made what was once a terminal illness into a treatable chronic condition. However, in low- and middle-income countries this development is notyet a reality for approximately $70 \%$ in a universe of 9.7 million of people estimated to be in need of ART but who do not access it. ${ }^{1}$ Among these countries, Mozambique is among those with enormous numerical need for ART coverage, with 280,000 patients clinically eligible to receive ART of whom less than $30 \%$ were receiving it up to the end of 2007. ${ }^{1}$ Identifying what is keeping the ART coverage rate very low is imperative, especially when the costs of antiretrovirals no longer seem to be a constraint, as these are provided for free.

A number of studies in low- and middle-income countries identified factors such as high costs, lack of information about ART, and stigma as factors that impede patients from accessing ART. ${ }^{2}$ Although these studies provide an important insight in the range of possible barriers they face a number of shortcomings. First, barriers are context-specific and results cannot be directly extrapolated. Second, little has been done in terms of comparing factors that impede patients from accessing ART in urban and in rural settings, while in these countries, these settings have different characteristics ranging from disparities in infrastructure to health service availability. Such disparities may affect differently patients' access to ART. Third, few studies used a combination of qualitative and quantitative methods, while a combination of in-depth understanding and structured knowledge is useful.

Against this background, this article aims to identify, rank, and compare factors perceived as barriers to accessing ART in urban and rural settings in Mozambique, on the basis of focus group discussions and large surveys among patients and health workers. In addition, the article aims to compare the perceptions of patients who were on ART with perceptions of those who had discontinued ART. Such information is useful to policy makers in designing interventions to reduce the barriers, while taking into consideration the setting, as barriers are often context-specific and should as such be carefully considered.

\footnotetext{
${ }^{1}$ Faculty of Economics and Management, Catholic University of Mozambique, Beira, Mozambique.

${ }^{2}$ Department of Public Health, Radboud University Nijmegen Medical Centre, Nijmegen, The Netherlands.

Both authors contributed equally to this development of this work.
} 


\section{Methods}

\section{Study area}

Mozambique has a population of approximately 21 million inhabitants $^{3}$ with an HIV/AIDS prevalence rate of $16 \%$ among adults (ages 15-49). The study was performed in the districts of Beira and Buzi, an urban and rural area, respectively, both located in the central region of Mozambique, the country's region with the second highest HIV/AIDS prevalence rate $(18 \%){ }^{4}$

\section{Setting and participants}

The study population consisted of people living with HIV/AIDS (PLWHA) and health workers. The PLWHA were included in this study if they were 18 years of age or older. As for the health workers, they were included in the study if they were doctors, clinic officers, nurses, laboratory technicians, social assistants, peer counselors (activists), and administrative personnel working in the antiretroviral program at the health facilities selected for the study. No specific demographics were collected from the health workers, apart from gender. In addition, participants were included only if they were willing to participate.

The PLWHA were recruited via HIV / AIDS support groups using nonrandom sampling and via the health facilities providing antiretrovirals, using random sampling. PLWHA recruited via HIV/AIDS support groups participated in the focus group discussions in the first phase of data collection while those recruited via health facilities participated in the interviews in the second phase. We used two sampling sources because focus group discussions require that participants interact and discuss their perceptions and experiences. According to the ethical committee, focus group discussions could only be performed with people who had disclosed their HIV positive status to others, in order to avoid exposing the positive status of people who had not yet disclosed their positive status. Therefore, we could only recruit people for the focus group discussions via the support groups were the positive status was known among them. For the second phase of data collection, we only recruited at the health facilities to avoid double-counting because most PLWHA in support groups were registered at the health facilities.

The PLWHA recruited via health facilities were located by peer counselors (working at the health facilities) who described the study to them, obtained consent and acted as translators during the interviews. The peer counselors received training in the research protocol, which involved educating them as to the nature of the research and the use of a standardized script with which to enroll participants. Before the focus group discussions and interviews, we ensured that participant's informed consent had been obtained.

Informed consent was obtained in writing (for those who did not mind giving their signature) and by fingerprints (for those who did mind). Participants received no remuneration for participating in the study. Patients who preferred to be interviewed at the health facility or at a different location instead of at their houses received a refund for transport costs for the return trip. They were informed about the objectives and purpose of the study, duration of the focus group discussions, and of the interviews. The research was approved by the Ethical Committee of the Catholic University of Mozambique.

\section{Data collection}

We collected data in two phases between March and July 2008. In the first phase we collected data through 13 focus group discussions, 7 with PLWHA and 6 with health workers. Each focus group discussion was composed of 8 to 12 members. The focus group discussion started with the following initial question: What makes it difficult for you to initiate or continue on antiretroviral treatment? For health workers, the question was framed differently: What makes it difficult for the patients to initiate or continue on antiretroviral treatment? The subsequent questions were based on the participants' contribution in the discussion. The focus group discussions were held separately for PLWHA who had initiated and/or discontinued ART and PLWHA who had not yet initiated ART. The focus group discussions for health workers were also held separately. We used this approach in order to enable each group to feel comfortable to contribute in the discussion. All focus group discussions were tape recorded.

The data from the focus group discussions provided the framework for the structured questionnaire used in the second phase of data collection. This questionnaire was administered face-to-face to 252 patients and 28 health workers in 2 health facilities ( 1 in Beira and 1 in Buzi). The questionnaire was composed of 63 statements. For each statement, the respondents could express their opinion on a five-point Likert scale: strongly disagree (0), disagree (1), neutral (2), agree (3), and strongly agree (4). The focus group discussions and questionnaire were administered in Portuguese, Sena, and Ndau (official and local languages in the study areas). The interviews were confidential, anonymous, and in a location of choice of the patient.

\section{Data analysis}

First, we categorized the perceptions of barriers to accessing ART elicited in the focus group discussions using content analysis. Content analysis is a procedure for the categorization of verbal or behavioral data, for purposes of classification, summarization and tabulation. ${ }^{5}$ During the focus group discussion, one person conducted the focus group discussion, one took notes, and one recorded the discussion. After each focus group discussion, a report was elaborated including the data from the three sources. After all focus group discussions were conducted, an analysis of the perceptions elicited was performed. Perceptions that referred to the same topic were put together and a name was given to each category. In this analysis three major categories of perceptions emerged, namely: (1) patient beliefs and attitudes, (2) health personal practice and conduct, and (3) availability and adequacy of resources. The items in each category were later tested in the questionnaire.

Second, we used factor analysis to analyze the data collected through the structured questionnaire. Factor analysis is a technique for identifying groups or clusters of variables, with the purpose of reducing a dataset to a more manageable size while retaining as much of the original information as possible. ${ }^{6}$ As for the particular method for factor analysis we used the principal component analysis (PCA) with Varimax rotation, and a factor loading cutting point of 0.5 . We extracted the factors based on the scree plot (Fig. 1) and confirmed the solution with the criterion of Eigenvalues greater than 1 . To ensure suitability of data for factor analysis, we evaluated the 
sample size adequacy using the Kaiser-Meyer-Olkin (KMO) for both multiple and individual variables. The overall KMO was computed at 0.71 and the KMO for individual variables ranged from above 0.5 , and these are considered satisfactory. ${ }^{6}$ The Bartlett's test of Sphericity tests the overall significance of all correlations in a correlation matrix, and it was significant at $p<0.0001$ level. For the factor analysis we used SPSS (version 16.0 for Windows, SPSS Inc., Chicago, IL).

Finally, we calculated the percentages of the maximum attainable scores for each item of the factors that resulted from the factor analysis, in order to rank them according to their relative importance and to compare them among the three groups, i.e., perceptions of patients in urban areas with perceptions of those in rural areas, perceptions of patients who were on ART with perceptions of those who had discontinued ART, and perceptions of patients with perceptions of health workers.

We calculated the percentages of the maximum attainable scores in the following way: in a scale of 0 to 4 , from strongly disagree to strongly agree, we multiplied the responses for each grade $(0,1,2,3$, and 4$)$ in the scale by the corresponding grades to get the scores. Then we multiplied the sum of the responses by the maximum grade (4) to get the maximum score. Then we divided the sum of the score by the maximum score to get the percentage of the maximum attainable score.

\section{Results}

\section{Characteristics of respondents}

Demographic data is summarized in Table 1. Most patients were in the 30 - to 49 -year age range (59\%). The majority of patients $(73 \%)$ in the study were women. $60 \%$ were separate, divorced, or widowers. Most patients (57\%) had primary education, and about $30 \%$ had no formal education. Most patients were unemployed $(93 \%)$. The majority of patients (81\%) were on ART, while $14 \%$ were not yet on ART and 5\% had discontinued ART. Of the 28 health workers interviewed, $64 \%$ were male and $36 \%$ were female. Their occupations ranged from doctors, nurses, social assistants, laboratory technicians, and peer counselors.

\section{Factors associated with access to ART}

Using principal component analysis with Varimax rotation, the 63 items in the questionnaire were reduced to 6 clusters of

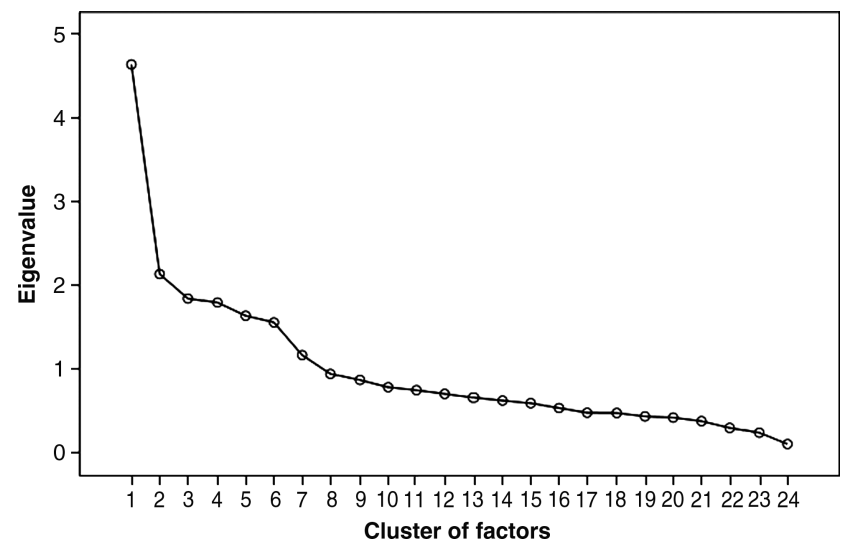

FIG. 1. Cluster of factors and eigenvalues. factors with 21 factors in total. Table 2 presents the rotated solution with factor loadings. Each cluster of factors had an Eigenvalue greater than 1.0 and with the cumulative variance at $54 \%$. The 6 clusters of factors were named as follows: (1) patient information and attitudes toward treatment, (2) health personnel confidentiality, (3) family support, (4) service availability, (5) community information, and (6) patient resource availability. Cluster one (patient information and attitudes toward treatment), accounted for approximately $11 \%$ of the total variance, with 6 factors on the acceptability of side effects of antiretrovirals, acceptability in changing antiretroviral lines, adequacy of information about antiretrovirals, adequacy of being seen at the day hospital, and adequacy of knowledge peer counselors have about antiretrovirals and HIV/AIDS. Cluster two (health personal confidentiality), accounted for approximately $9 \%$ of the variance, with 2 factors on confidentiality. Cluster three (family support), accounted for approximately $9 \%$ of the variance with 4 factors on confidentiality by relatives, encouragement by relatives in seeking antiretrovirals, discrimination by relatives, and disclosure to relatives. Cluster four (service availability), accounted for approximately $9 \%$ of the variance with 4 factors related to the waiting time to receive results of CD4 (cluster of differentiation 4) and biochemistry (hemoglobin and liver tests) analysis, the waiting time to see a doctor, and the sufficiency of doctors/nurses at the health facility. Cluster five (community information), accounted for approximately $8 \%$ of the variance, with 2 factors on the adequacy of information about ARVs and HIV/AIDS in the community. Cluster six (patient resource availability), accounted for approximately $7 \%$ of the variance, with 3 factors on the availability of transportation and food, and adequacy of distance to the health facility. Table 3 contains information relating to each cluster of factor's variance and Eigenvalue.

\section{Ranking factors associated with access to ART}

We used the percentages calculated through the maximum attainable scores to rank the cluster of factors according to their relative importance. The clusters of factors with percentages below 50\% were considered barriers to accessing ART. In general, patients and health workers were very negative about patient resource availability, rating in this cluster the distance from home to the health facility, transportation, and food availability below $40 \%$ of the maximum attainable score. This cluster was followed by clusters of factors on community information rated at $47 \%$ and by service availability rated at $53 \%$ in which the waiting time to receive the results of CD4 analysis and the sufficiency of doctors/ nurses at the health facility were both rated at $45 \%$. The other cluster of factors were rated positively, i.e., patient information and attitudes toward treatment (74\%), family support $(77 \%)$, and health personnel confidentiality $(79 \%)$.

\section{Comparison of factors associated with access to ART among the different groups}

The comparison of factors associated with access to ARTs was between urban and rural patients, patients on ART and patients who have discontinued ART, and between patients and health workers.

Both urban and rural patients were relatively positive about patient information and attitudes toward treatment 
Table 1. Respondents Characteristics

\begin{tabular}{|c|c|}
\hline Patients $(\mathrm{n}=252)$ & No $(\%)$ \\
\hline \multicolumn{2}{|l|}{ Age (years) } \\
\hline $18-29$ & $78(31.3)$ \\
\hline $30-49$ & $148(59.4)$ \\
\hline$\geq 50$ & $23(9.2)$ \\
\hline \multicolumn{2}{|l|}{ Gender } \\
\hline Female & $185(0.74)$ \\
\hline Male & $67(0.27)$ \\
\hline \multicolumn{2}{|l|}{ Marital status } \\
\hline Single, divorced, widower & $152(60.3)$ \\
\hline Married & $100(39.7)$ \\
\hline \multicolumn{2}{|l|}{ Level of education } \\
\hline No formal education & $76(30.3)$ \\
\hline Primary education & $142(56.6)$ \\
\hline Secondary education & $21(8.4)$ \\
\hline Postsecondary education & $12(4.8)$ \\
\hline \multicolumn{2}{|l|}{ Employment status } \\
\hline Employed & $17(6.75)$ \\
\hline Unemployed & $235(93.25)$ \\
\hline \multicolumn{2}{|l|}{ Status of $A R V s$} \\
\hline Not yet on ARVs & 35 (13.9) \\
\hline On ARVs & $204(80.9)$ \\
\hline Discontinued ARVs & $11(5.1)$ \\
\hline \multicolumn{2}{|l|}{ Health personnel $(\mathrm{n}=28)$} \\
\hline \multicolumn{2}{|l|}{ Gender } \\
\hline Female & $10(35.7)$ \\
\hline Male & $18(64.3)$ \\
\hline \multicolumn{2}{|l|}{ Occupation } \\
\hline Doctor & $1(3.57)$ \\
\hline Nurse & $9(32)$ \\
\hline Social assistant & $3(10.7)$ \\
\hline Laboratory technician & $2(7.14)$ \\
\hline Peer counsellors & $13(46.4)$ \\
\hline
\end{tabular}

ARVs, antiretrovirals.

( $82 \%$ versus $69 \%$ ), health personnel confidentiality (82\% versus $76 \%$ ) and family support (79\% versus $75 \%)$. Urban patients were relatively positive about service availability while rural patients were rather negative ( $59 \%$ versus $48 \%$ ). Both groups were negative regarding community information ( $45 \%$ versus $49 \%$ ) and very negative about patient resource availability (39\% versus 35\%). In general, rural patients' ratings were lower than urban patients' ratings $(0.64 \%$ versus $0.59 \%)$.

As for the comparison of perceptions of patients who were on ART and those who had discontinued ART, both groups were positive about patient information and attitudes toward treatment (77\% versus $83 \%)$, health personnel confidentiality (78\% versus $75 \%$ ), and family support ( $80 \%$ versus $83 \%$ ). Patients who were on ART were less positive about service availability than patients who had discontinued ART, rating negatively the waiting time to receive the results of CD4 $(48 \%$ versus $64 \%$ ) and the sufficiency of doctors/nurses in the health facility ( $47 \%$ versus $55 \%$ ). Patients who were on ART were also negative regarding community information $(47 \%$ versus $56 \%$ ). As for patient resource availability both groups rated most of the factors in this cluster negatively, with the exception of availability of transportation, which was rated positively by patients who had discontinued ART $(0.37 \%$ versus $0.59 \%$ ). Overall, ratings of patients who were on ART were lower than the ratings of patients who had discontinued ART $(0.63 \%$ versus $0.69 \%)$.

Regarding the comparison of patients and health workers, the cluster of factors rated positively and negatively by one group were also rated as such by the other group, except in the third cluster (family support), where health workers rated the disclosure of HIV status to relatives negatively (43\% versus $74 \%$ ). Within the factors rated negatively by both groups, there were large differences. In service availability health workers rated further negatively the waiting time to receive the CD4 analysis ( $24 \%$ versus $47 \%$ ), the sufficiency of doctors/ nurses in the health facility ( $29 \%$ versus $47 \%$ ), the availability of food ( $26 \%$ versus $40 \%)$, and the distance from home to the health facility (26\% versus $38 \%$ ) (Table 4 ).

\section{Discussion}

We identified patient information and attitudes toward treatment, health personnel confidentiality, family support, service availability, community information, and patient resource availability as clusters of factors associated with access to ART. While respondents were positive about the cluster on patient information and attitudes toward treatment, health personnel confidentiality and family support, they were negative about the cluster on patient resource availability, community information and two factors in the cluster on service availability. These clusters of factors may be considered barriers to accessing ART as they may deter overall access, and are discussed in more detail below.

First, patients were very negative about patient resource availability, particularly on the availability of transportation and food and on the adequacy of distance from the house to the health facility. These factors appear to be the most important barriers to access and have also been found as barriers in several studies. ${ }^{7-11}$ The problem of transportation and distance is most of the times interlinked, since the distance to health facilities imposes considerable cost on patients and this may deter access. ${ }^{9}$ Given that $93 \%$ of the patients interviewed in our study (Table 1) were unemployed; lack of financial means may thus explain why the above may constitute barriers to access. While on one hand the problem of transportation and distance is merely a demand problem, on the other hand, it may be a supply problem. Transportation costs are usually high in settings where public transportation is not well developed and that HIV/AIDS services may not be near communities. Since the development of public transportation is beyond the scope of the health sector, an option to address these barriers could be the provision of HIV/AIDS services and ART near communities, as this may reduce distances travelled by patients and costs associated to it. Mobile clinics could be an example of such a strategy.

Second, patients were negative concerning adequacy of information about HIV/AIDS and ARVs in the community. This confirms findings from Zambia, where it was found that communities lacked information on HIV/AIDs and on ARVs. ${ }^{12,13}$ On the light of this, policy makers may pay particular attention to the dissemination of information about HIV/AIDS and ART in communities, since the lack of it is considered a drive of stigma and discrimination that is often attached to HIV/AIDS. ${ }^{14}$ And stigma could interfere with sustained participation at all stages of the treatment 


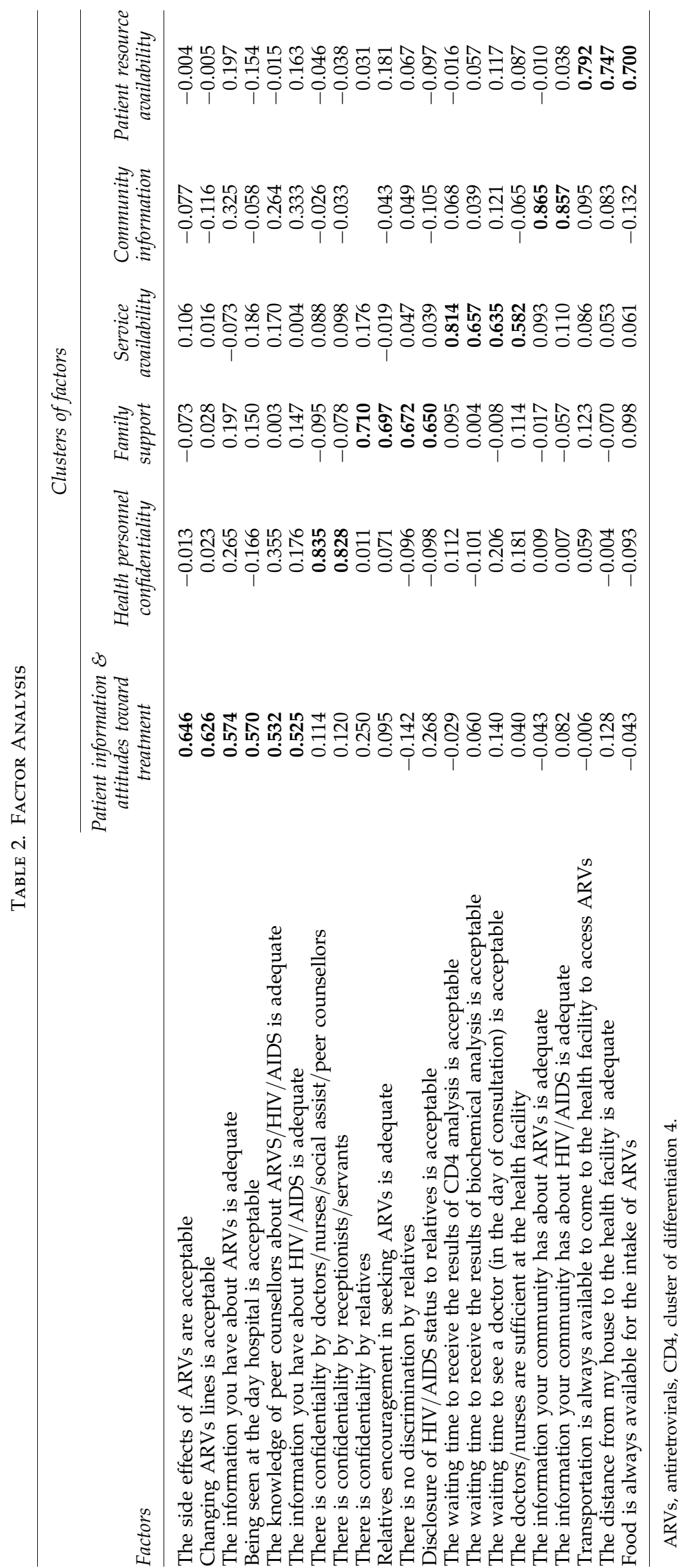


TABle 3. Eigenvalues ANd Factors Variance

\begin{tabular}{lccc}
\hline Cluster of factors & Eigenvalues & \% of variance & Cumulative \% of variance \\
\hline Patient information and attitudes toward treatment & 3.09 & 11.45 & 11.45 \\
Health personnel confidentiality & 2.56 & 9.46 & 20.91 \\
Family support & 2.51 & 9.30 & 30.21 \\
Service availability & 2.48 & 9.19 & 39.40 \\
Community information & 2.08 & 7.71 & 47.11 \\
Patient resource availability & 1.96 & 7.27 & 54.37 \\
\hline
\end{tabular}

continuum-from discouraging voluntary counseling and testing and sharing results, to causing patients to hide their treatment-which could prove difficult over time and thereby threaten adherence. ${ }^{8}$ An additional implication of inadequacy of information about ART could be the increased risk of HIV infection as found in a population-based study in Kenya. ${ }^{15}$ In this study the majority of participants had heard of ART and knew some basic information about it, but a sizeable minority had misinformation that ART cured HIV/AIDS and believed that they were now free to engage in risky behavior.

Third, rural patients were relatively negative on the waiting time to receive the results of CD4 analysis. This could be related to the fact that in Mozambique for example, most rural health facilities do not have conditions to perform a CD4 analysis. Therefore they have to send the blood samples to labs in health facilities in urban areas, and this may take more time than it would take if the analysis were performed in the same place. Waiting times have also been found in other studies as a reason for patient dissatisfaction when seeking ART services. ${ }^{10,16}$ Rural patients also rated negatively the sufficiency of doctors/nurses, and this could also be explained for instance by the extremely high patient load of a rural physician (approximately 6500 patients) compared to a urban physician patient load (approximately $300 \mathrm{HIV}$-positive patients) in Mozambique. ${ }^{17}$ The need to increase physicians and other health care workers especially in rural settings is once more evident. Policy makers need to urgently develop interventions to mobilize, retain, and train health workers. Recruitment of retired health workers is an example of an intervention that could increase the numbers of health workers without creating a gap elsewhere in the health system. For example, an HIV/AIDS reduction and prevention project in Guyana addressed the shortage of health workers by recruiting retired nurses. ${ }^{18}$ Including community health workers in the provision of HIV/AIDS services ${ }^{19}$ and the provision of scholarships for health care education in return for a commitment to deliver ART are other options. ${ }^{20}$

A number of further observations could be made in relation to the factors rated negatively. Overall, patients who were on ART were less favorable than patients who had discontinued ART. They rated almost all items lower than the patients who had discontinued ART. The patients who had discontinued ART only rated negatively the adequacy of the distance from home to the health facility and the availability of food. This may indicate that these constitute the main reasons for discontinuation of ART among these patients. Other studies have also found transport costs and inadequate nutrition as factors posing the most intractable barriers to sustaining ART. ${ }^{10,11}$ Discontinuation of ART raises concerns of drug resistance, because once started, ART should be taken for the rest of one's life and these factors pose a threat for the long-term retention of patients in treatment programs. ${ }^{21}$ In reducing these barriers policy makers may ensure that patients who initiate ART continue for the rest of their lives, thereby achieving the goal of improving the quality and life expectancy of people living with HIV/AIDS. Both patients and health workers rated similarly all the factors, with an exception of factor three (family support), where health workers rated the disclosure of HIV status to relatives negatively.

A number of clusters of factors were rated positively. The cluster rated most positively was health personnel confidentiality. Confidentiality could be interpreted as a component of trust. A trusting relationship between patients and health workers is important as it increases initiation, adherence and continuation of patients into treatment. ${ }^{23,24}$ In the literature there are mixed evidences, with studies reporting findings similar ${ }^{10,22}$ and contradictory to our findings. ${ }^{25}$

The second cluster of factors rated positively was patient information and attitudes toward treatment. This could be interpreted as an indicator that the information provided at the health facility is understood by patients, since most patients in our study were on ART, and all had been in contact with the health facility. A similar finding has been found in a study in South Africa which demonstrated that HIV clinic patients had a good knowledge of HIV/AIDS and ART. ${ }^{26}$ It is believed that good knowledge of treatment and disease among patients may lead to a positive attitude toward ART, such as acceptance of treatment and of side effects.

The third cluster of factors rated positively was family support. This finding supports similar results regarding the role of family in patient continuation into ART. ${ }^{11,27}$ However, our study did not specifically identify financial support as identified in those studies. In our study, most of the factors in the family support cluster refer to confidentiality, discrimination, and disclosure, with an exception of one that refers to the encouragement of relatives in seeking ART. It is possible that in this factor the financial aspect is included. The factor on acceptability of disclosure of the HIV status to relatives was rated positively by patients but negatively by health workers. The negative rating by health workers may be explained by their perception of the stigma patients experience from relatives, as found in other studies. ${ }^{25,26}$ While the positive rating of the acceptability of disclosure of the HIV status to relatives by patients may be explained by the influence of the community support groups to which most patients are members and other aspects such as counseling, information, education, and by the strength of their faith. ${ }^{25,29}$ In addition, a study in Ethiopia also suggested that the disclosure of HIV positive status by patients was high and that relatives were the first ones to whom patients disclosed their status to. ${ }^{28}$ 


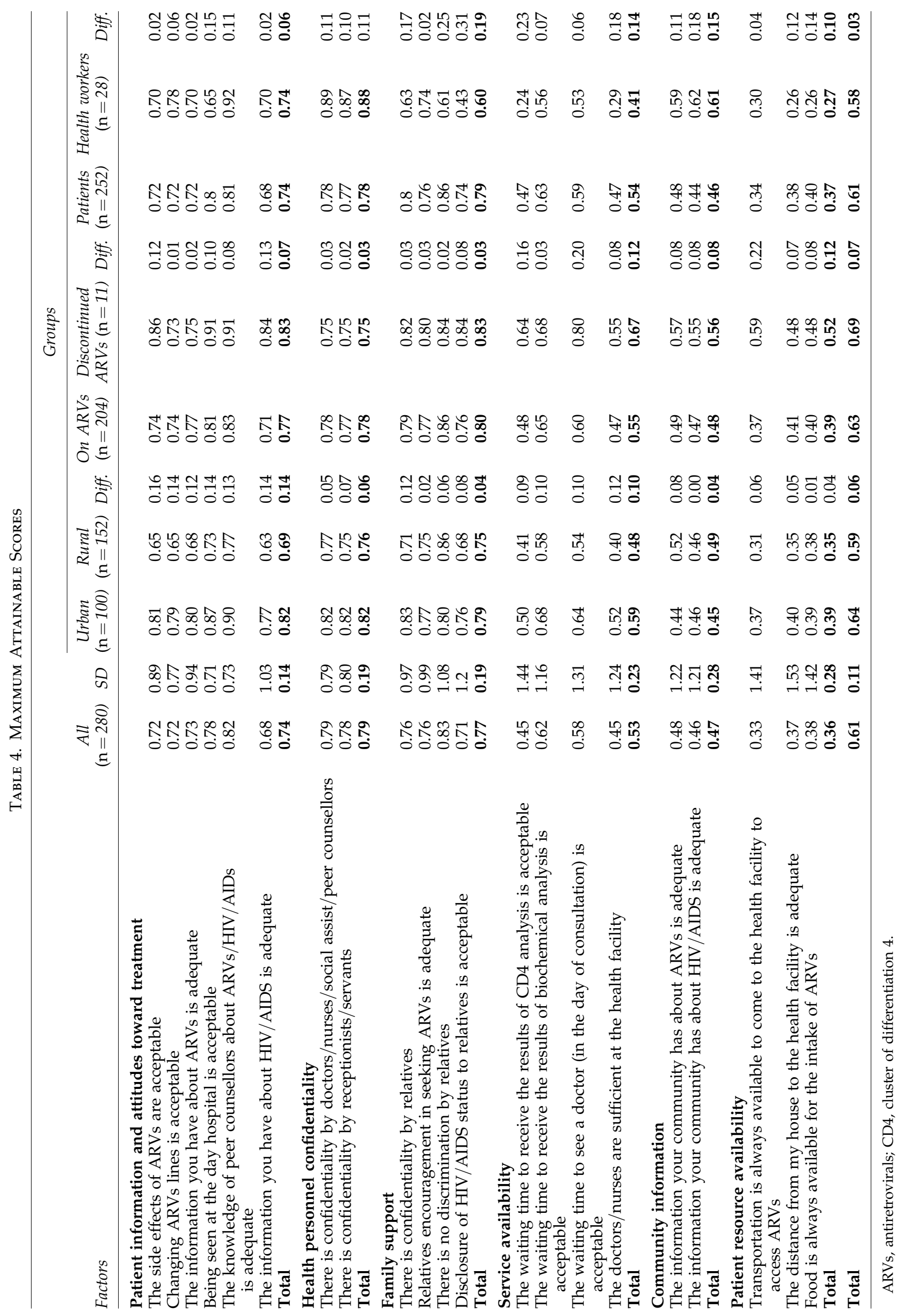


In our study we used a combination of qualitative and quantitative methods to collect data. The use of focus group discussions in phase one enabled the identification of relevant factors that were later tested using a questionnaire. This combination of methods as well as the involvement of patients and health workers, allowed a cross-validation of data and possibly a minimization of bias, thereby constituting strength of our study.

Our study is not without limitations. We worked with peer counselors during the data collection stage. Although this was a strength in the sense that it was useful to locate patients and to establish trust between the interviewers and the patients, it could also be considered a limitation as it may have lead patients to give favorable answers, in particular to the questions related to health system factors. We could not avoid the presence of peer counselors because they were the ones who knew the exact residence address of the patients. The other reason we worked with them was that we needed translators for the interviews, especially on rural areas and we could not request anyone outside the health facility, in order to preserve the confidentiality and privacy of the patients.

The relatively small sample size of patients who had discontinued ART (11) could also be considered a limitation, as it could affect the confidence with which these findings can be generalized for this group. Future research could use larger numbers of patients who have discontinued ART. It was difficult to locate patients who had discontinued ART because some were found to be dead when traced, some had already reinitiated ART, and others had changed their residence address.

Another limitation of our study is that we did not differentiate between those patients who had not initiated ART because of not being eligible to receive ART and those who had not initiated because of other reasons. Therefore, we could not compare their perceptions in terms of barriers to access to ART. Future research could address this and thereby provide additional information regarding this group.

Despite the shortcomings, our study offers one of the first empirical insights into the barriers to access to ART in Mozambique's urban and rural areas as perceived by HIV/AIDS patients and health workers. Policy makers, in efforts to further improve the access to ART may decide to target their attention in designing interventions to improve specific aspects of patient resource availability, community information, and service availability in both urban and rural settings in Mozambique.

\section{Acknowledgments}

We would like to thank the supporting groups of people living with HIV/AIDS and the health facilities for their collaboration. We would also like to thank the persons who participated in the study. In addition, we would like to thank Dr. Cristiano Matsinhe for his comments on the design of the study and Irvan Afriandi for his technical assistance.

This study was funded by NUFFIC (The Netherlands Organization for International Cooperation in Higher Education) under the Research and Education program on HIV/AIDS in Mozambique (NUFFIC, NPT/MOZ/083). The funder had no role in study design, data collection and analysis, decision to publish, or preparation of the manuscript.

\section{Author Disclosure Statement}

No competing financial interests exist.

\section{References}

1. WHO/UNAIDS/UNICEF. Towards Universal Access: Scaling Up Priority HIV/AIDS Interventions in the Health Sector. Progress Report 2008. Geneva: World Health Organization, 2008.

2. Posse M, Meheus F, van Asten $H$, van der Ven A, Baltussen R. Barriers to access to antiretroviral treatment in developing countries: A review. J Trop Med Int Health 2008;13:904-913.

3. United Nations. 2010 World Population and Housing Census Programme. http://unstats.un.org/unsd/demographic/ sources/census/censusdates.htm (Last accessed October 1, 2008).

4. Mozambique Progress Report for the United Nations General Assembly. Special Session on HIV/AIDS 2008. http://data .unaids.org/pub/Report/2008/mozambique_2008_country_ progress_report_en.pdf (Last accessed September 5, 2008).

5. Hancock B. Trent Focus for Research and Development in Primary Health Care: An Introduction to Qualitative Research. Nottingham, UK: Trent Focus, 2002. www.trentrdsu .org.uk/cms/uploads/Qualitative\%20Research.pdf (Last accessed September 5, 2008).

6. Field A. Exploratory factor analysis. In: Discovering Statistics Using SPSS, 2nd ed. London: Sage, 2005:619-666.

7. Ensor T, Cooper S. Overcoming barriers to health service access: Influencing the demand side. Health Policy Plan 2000;19:69-79.

8. Mshana GH, Wamoyi J, Busza J, et al. Barriers to accessing antiretroviral therapy in Kisesa, Tanzania: A qualitative study of early rural referrals to the national program. AIDS Patient Care STDs 2006;20:649-657.

9. Zachariah R, Harries DA, Manzi M, et al. Acceptance of antiretroviral therapy among patients infected with HIV and tuberculosis in rural Malawi is low and associated with cost of transport. PLoS ONE 2006;1:e121.

10. Hardon AP, Akurut D, Comoro $C$, et al. Hunger, waiting time and transport costs: Time to confront challenges to ART adherence in Africa. AIDS Care 2007;19:658-665.

11. Roura M, Busza J, Wringe A, Mbata D, Urassa M, Zaba B. Barriers to sustaining antiretroviral treatment in Kisesa, Tanzania: A follow-up study to understand attrition from the antiretroviral program. AIDS Patient Care STDs 2009;23: 203-210.

12. International AIDS Alliance. Voices from Zambian Communities: Experiences of HIV/AIDS related treatment in urban and rural settings. A report of community consultations on HIV/AIDS-related treatment. International AIDS Alliance, Lusaka, Zambia. 2003. www.synkronweb.aidsalliance.org/ graphics/secretariat/publications/cim1004_Voices_from_ Zambian_communities.pdf (Last accessed September 5, 2008).

13. Grant E, Logie D, Masura M, Gorman D, Murray A. Factors facilitating and challenging access and adherence to antiretroviral therapy in a township in the Zambian Copperbelt: A qualitative study. AIDS Care 2008;21:78-86.

14. Campbell C, Nair Y, Mailman S, Nicholson J. "Dying twice." A multi-level model of the roots of aids stigma in two South African communities. J Health Psychol 2007;12:403-416.

15. Cohen CR, Montandon M, Carrico AW, et al. Association of attitudes and beliefs towards antiretroviral therapy with 
hIV-seroprevalence in the general population of Kisumu, Kenya. PLoS ONE 2009;4:e4573.

16. Wouters E, Heunis C, van Rensburg D, Meulemans H. Patient satisfaction with antiretroviral services at primary health-care facilities in the Free State, South Africa-A twoyear study using four waves of cross-sectional data. BMC Health Serv Res 2008;8:210.

17. Hagopian A, Micek MA, Vio F, Gimbel-Sherr K, Montoya P. What if we decided to take care of everyone who needed treatment? Workforce planning in Mozambique using simulation of demand for HIV/AIDS care. Hum Resourc Health $2008 ; 6: 1-9$.

18. Morgan R. Addressing health workers shortage: Recruiting retired nurses to reduce mother to child transmission in Guyana. Family Health International. 2005 http://pdf.usaid .gov/pdf_docs/PNADJ274.pdf (Last accessed October 18, 2008).

19. Mukherjee JS, Eustache FRE. Community health workers as a cornerstone for integrating HIV and primary health care. AIDS Care 2007;19:S72-S82.

20. Bärnighausen T, Bloom DE. "Conditional scholarships" for HIV/AIDS health workers: Educating and retaining the workforce to provide antiretroviral treatment in Sub-Saharan Africa. Soc Sci Med 2009;68:544-551.

21. Rosen S, Fox MP, Gill CJ. Patient retention in antiretroviral therapy programs in sub-Saharan Africa: A systematic review. PLoS Med 2007;4:e298.

22. Preau M, Leport C, Villes V, et al. Prevalence and predictors of deterioration of a trustful patient-provider relationship among HIV-infected persons treated with antiretroviral therapy. J Acquir Immune Defic Syndr 2008;47:467-471.

23. Mallinson RK, Rajabiun S, Coleman S. The provider role in client engagement in HIV care. AIDS Patient Care STDs 2007;21:S77-S84.
24. Whetten K, Leserman J, Whetten R, et al. Exploring lack of trust in care providers and the government as a barrier to health service use. Am J Public Health 2006;96:716-721.

25. Greeff M, Phetlhu R, Makoae LN. Disclosure of HIV status: Experiences and perceptions of persons living with hiv/aids and nurses involved in their care in Africa. Qual Health Res 2008;18:311-324.

26. Nachega JB, Lehman DA, Hlatshwayo D, Mothopeng R, Chaisson RE, Karstaedt AS. HIV/AIDS and antiretroviral treatment knowledge, attitudes, beliefs, and practices in HIV-infected adults in Soweto, South Africa. J Acquir Defic Syndr 2005;38:2.

27. Ware NC, Idoko J, Kaaya $S$, et al. Explaining adherence success in sub-Saharan Africa: An ethographic study. Plos Med 2009;6:0039-0047.

28. Deribe K, Woldemichael K, Wondafrash M, Haile A, Amberbir A. Disclosure experience and associated factors among HIV positive men and women clinical service users in southwest Ethiopia. BMC Public Health 2008;8:81.

29. Ncama BP. Acceptance and disclosure of HIV status through an integrated community/home-based care program in South Africa. Int Nurs Rev 2007;54:391-397.

Address correspondence to: Mariana Posse, M.A. Faculty of Economics and Management Catholic University of Mozambique Av. Eduardo Mondlane, 960 Beira Sofala 821 Mozambique

E-mail: possemariana@gmail.com 
